\title{
Impact of COVID-19 on Public Mental Health and the Buffering Effect of a Sense of Coherence
}

\author{
Sarah K. Schäfer ${ }^{\mathrm{a}}$ M. Roxanne Sopp ${ }^{\mathrm{a}} \quad$ Christian G. Schanz $^{\mathrm{a}}$ \\ Marlene Staginnus $^{b}$ Anja S. Göritz ${ }^{c}$ Tanja Michael ${ }^{a}$ \\ ${ }^{a}$ Clinical Psychology and Psychotherapy, Saarland University, Saarbruecken, Germany; \\ ${ }^{\mathrm{b}}$ Department of Psychology, University of Bath, Bath, UK; ${ }^{\mathrm{C}}$ Department of Occupational and \\ Consumer Psychology, Freiburg University, Freiburg, Germany
}

\section{Keywords}

COVID-19 - Mental health · Sense of coherence .

Psychopathology · Prospective

\begin{abstract}
Introduction: It is claimed that the coronavirus disease 2019 (COVID-19) pandemic has had a negative impact on mental health. However, to date, prospective studies are lacking. Moreover, it is important to identify which factors modulate the stress response to the pandemic. Previously, sense of coherence (SOC) has emerged as a particularly important resistance factor. Objective: This prospective study aimed to assess the impact of the COVID-19 outbreak on mental health and to investigate the ability of pre-outbreak SOC levels to predict changes in psychopathological symptoms. Methods: This study assessed psychopathological symptoms and SOC before and after the COVID-19 outbreak as well as postoutbreak COVID-19-related traumatic distress in a Germanspeaking sample $(n=1,591)$. Bivariate latent change score (BLCS) modeling was used to analyze pre- to post-outbreak changes in psychopathological symptoms and the ability of SOC to predict symptom changes. Results: Overall, there was no change in psychopathological symptoms. However,
\end{abstract}

on an individual-respondent level, 10\% experienced a clinically significant increase in psychopathological symptoms and $15 \%$ met cut-off criteria for COVID-19-related traumatic distress. Using BLCS modeling, we identified a high-stress group experiencing an increase in psychopathological symptoms and a decrease in SOC and a low-stress group showing the reversed pattern. Changes in SOC and psychopathological symptoms were predicted by pre-outbreak SOC and psychopathological symptom levels. Conclusions: Although mental health was stable in most respondents, a small group of respondents characterized by low levels of SOC experienced increased psychopathological symptoms from pre- to post-outbreak. Thus, SOC training might be a promising approach to enhance the resistance to stressors.

C 2020 The Author(s)
Published by S. Karger AG, Basel

\section{Introduction}

In March 2020, the outbreak of the coronavirus disease 2019 (COVID-19) reached all countries of the Western world [1]. To reduce the speed of its spread, many countries slowed down their economies and enforced pronounced restrictions on public life. karger@karger.com www.karger.com/pps

Karger $\stackrel{\text { ' }}{5}$

GOPEN ACCESS (c) 2020 The Author(s)

Published by S. Karger AG, Basel

This is an Open Access article licensed under the Creative Commons Attribution-NonCommercial-4.0 International License (CC BY-NC) (http://www.karger.com/Services/OpenAccessLicense), applicable to the online version of the article only. Usage and distribution for commercial purposes requires written permission.
Prof. Tanja Michael

Clinical Psychology and Psychotherapy, Saarland University Building A 13

DE-66123 Saarbruecken (Germany)

t.michael@mx.uni-saarland.de 


\section{Effects of COVID-19 on Mental Health}

It is well known that pandemics pose a threat to mental health [2-4]. Correspondingly, 2 recent reviews found a consistently negative impact of COVID-19 on mental health, with $16-18 \%$ of participants showing symptoms of anxiety and depression $[5,6]$. The first evidence indicates that women $[7,8]$, younger people [9], and those with a poor sleep quality $[7,9]$ are at an increased risk for mental health problems.

However, so far, no study has assessed whether mental health in the general population has genuinely changed from pre- to post-outbreak. We had conducted a panel study in Germany shortly before the outbreak (February $17-23,2020)$ that investigated associations between mental health and health-promoting factors. We were able to contact respondents again in mid-March after the outbreak to gather data reflective of the pandemic's impact on mental health. Furthermore, we assessed the resistance factor of sense of coherence (SOC), which constitutes an important step towards developing interventions aimed at buffering the effects of global stressors [10-12].

\section{Potential Use of Resistance Factors}

Such interventions may target resistance factors like SOC, the key component of the salutogenesis framework $[13,14]$. Individuals with high levels of SOC perceive life as comprehensible and manageable and believe that life challenges reflect a potential source of growth. In the salutogenesis framework, SOC is conceptualized as a stable disposition that is particularly important in situations that are perceived as highly demanding [15] (see online suppl. material; for all online suppl. material, see www. karger.com/doi/10.1159/000510752). However, previous research has also found short-term stressor-related changes in SOC, challenging its temporal stability [16]. SOC shows a robust positive correlation with mental health [17-19]. Moreover, it may also reflect an aspect of euthymia $[20,21]$. The positive component of euthymia is characterized by resistance, flexibility, and a unifying outlook on life-guiding actions and feelings to shape one's future, the latter of which may be encompassed by SOC.

Yet, little is known about the ability of SOC to predict changes in mental health. Only 2 studies - assessing women after pregnancy loss and patients with mental disorders in a psychosomatic rehabilitation clinic - found SOC to be predictive of changes in mental health $[22,23]$. However, these studies did not apply state-of-the-art methods for prospective data [24].

Impact of COVID-19 on Public Mental Health

\section{Study Aim}

In the current study, we aimed to examine the number of respondents who experienced a clinically significant change in psychopathological symptom levels from preto post-outbreak assessment or significant levels of COVID-19-related traumatic distress. Based on previous studies on COVID-19-related traumatic distress [5, 25], we expected significant levels of traumatic distress in 10 $20 \%$ of the sample and stronger stress responses in females, younger respondents, and those reporting a poor sleep quality. Moreover, building on studies on the SARS epidemic [26], we hypothesized that some respondents would show an increase in psychopathological symptoms from pre- to post-outbreak. In line with previous research [16], we expected SOC to decrease over time in those experiencing high levels of stress. Second, we aimed to examine the ability of SOC to predict symptom changes, which should be particularly strong in those experiencing high COVID-19-related stress.

\section{Materials and Methods}

Study Design and Sample Recruitment

The current study is part of a larger project investigating the factorial structure of health-promoting factors. For sample recruitment, we used an online panel (WiSoPanel, https://www.wisopanel.net [27]; see online suppl. material for details). Data were collected via the platform SoSci Survey [28] and respondents gave written informed consent according to the Declaration of Helsinki [29] (Fig. 1; online suppl. material).

\section{Measures}

SOC was assessed using a 9-item short version of the Antonovsky scales [30]. Psychopathological symptoms were measured using the Mini-Symptom Checklist [31]. Sleep quality during the last week was measured at the post-outbreak assessment using the Single-Item Sleep Quality Scale [32]. COVID-19-related rumination was evaluated at the post-outbreak assessment using a modified version of the Perseverative Thinking Questionnaire (PTQ) [33]. COVID-19-related traumatic distress was measured using a modified version of the Peritraumatic Distress Inventory (PDI) [34]. Following Bunnell et al. [35], scores $\geq 23$ indicate a greater risk for COVID-19-related traumatic distress (see online suppl. material).

\section{Calculation of COVID-19-Related Stress Scores}

To assess respondents' COVID-19-related stress, we calculated a COVID-19 stress index as the sum of the $z$-standardized scores of PTQ and PDI (see online suppl. material). Higher scores indicate more severe stress. Scores $>0$ reflect an above-average COVID-19-related stress burden (high COVID-19-related stress group, score $>0$; low COVID-19-related stress group, score $\leq 0$ ). 


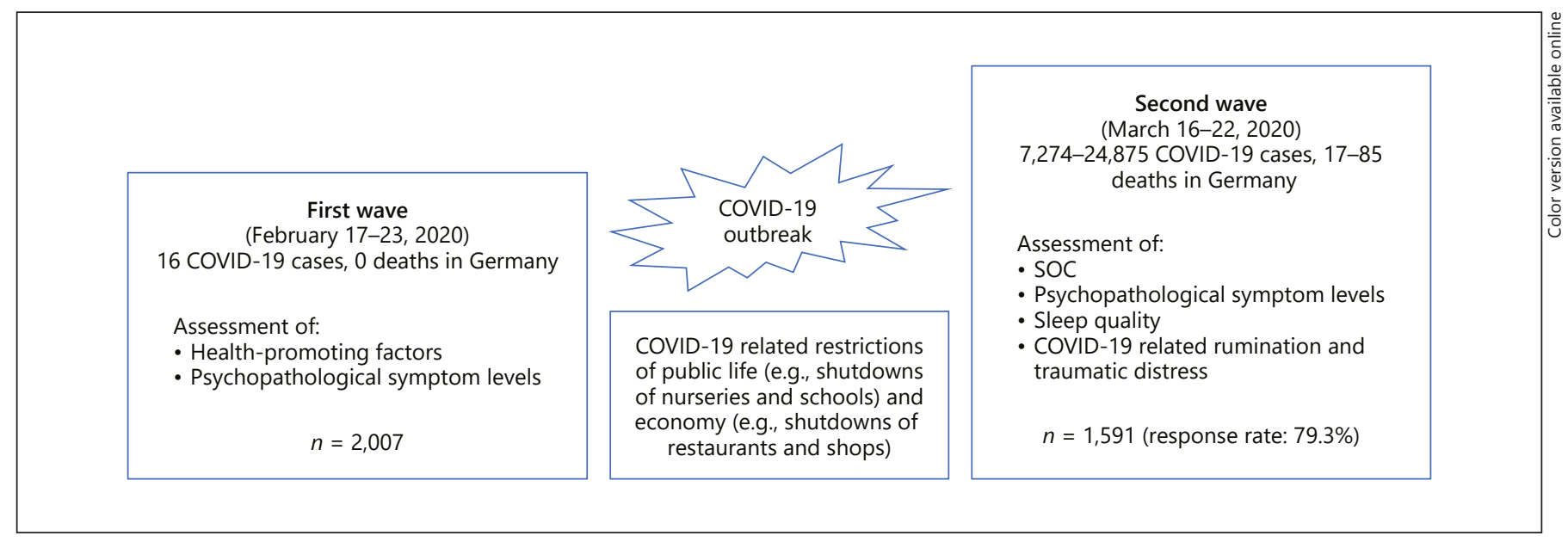

Fig. 1. Details of the study design.

Data Analyses

Analyses were conducted using RStudio [28] and the lavaan package [29]. We calculated reliable change indices according to the Mini Symptom Checklist (Mini-SCL) manual to quantify clinically significant changes in psychopathological symptoms. To further analyze pre- to post- changes in psychopathological symptoms and SOC and the ability of SOC to predict symptom changes, we applied bivariate latent change score (BLCS) models $[27,36]$ (see online suppl. material).

\section{Results}

\section{Sample Characteristics}

The mean age of the participants who completed the second assessment $(n=1,591)$ was 55.03 years $(\mathrm{SD}=$ 13.90, range: $20-95)$ and $53.6 \%$ were female. The majority lived in Germany (96.2\%), 2.3\% lived in Austria, 1.1\% lived in Switzerland, and 0.4\% were German speaking but reported living in other countries (i.e., French-German border area). See the online supplementary material for a comparison with the German general public and results after excluding respondents not living in Germany.

\section{Reliable Change in Psychopathological Symptoms}

We analyzed reliable change indices and found significant changes from pre- to post-outbreak in $18 \%$ of the respondents $(n=287)$, whereby $10 \%(n=152)$ showed a significant increase and $8 \%(n=135)$ a significant decrease in psychopathological symptom levels. Moreover, 15\% exceeded the PDI cut-off for traumatic distress.

\section{Pre- to Post-Outbreak Change Using BLCS}

The separate BLCS models for the total sample, the high-stress group (stress score $>0, n=634$ ) and the lowstress group (stress score $\leq 0, n=862$ ) showed a good fit $(\mathrm{CFI}=1.00 ; \mathrm{SRMR}=0.00)$. In the total sample, there was no change in psychopathological symptoms and SOC levels from pre- to post-outbreak (Fig. 2; Table 1). By contrast, in the high-stress group, symptom levels increased while SOC levels decreased. Changes in SOC levels and psychopathological symptoms were negatively correlated. In the low-stress group, psychopathological symptoms decreased from pre- to post-outbreak assessment and SOC levels increased. Again, both changes were negatively correlated.

\section{Differences between the High and Low-Stress Groups}

Analyses of group differences revealed that individuals in the high-stress group reported a poorer sleep quality $(t[1,469]=6.72, p<0.001, d=0.35, \mathrm{OR}=1.16)$ and were younger $(t[1,470]=3.74, p<0.001, d=0.20$; OR $=1.01)$ and more likely to be female $\left(\chi^{2}[1]=12.69, p<0.001\right.$, OR $=1.46)$. The groups did not differ in terms of education level $\left(\chi^{2}[5]=7.06, p=0.217\right)$. Moreover, the respondents in the high-stress group were not more likely to live in the federal states of Germany that had reported the highest numbers of COVID-19 cases at the post-outbreak assessment (i.e., Bavaria and North-Rhine Westphalia; $\chi^{2}[1]=$ $0.00, p=0.974, \mathrm{OR}=1.00$ ).

\section{Ability of SOC to Predict Symptom Changes}

In the total sample, individual changes in psychopathological symptoms were significantly predicted by 


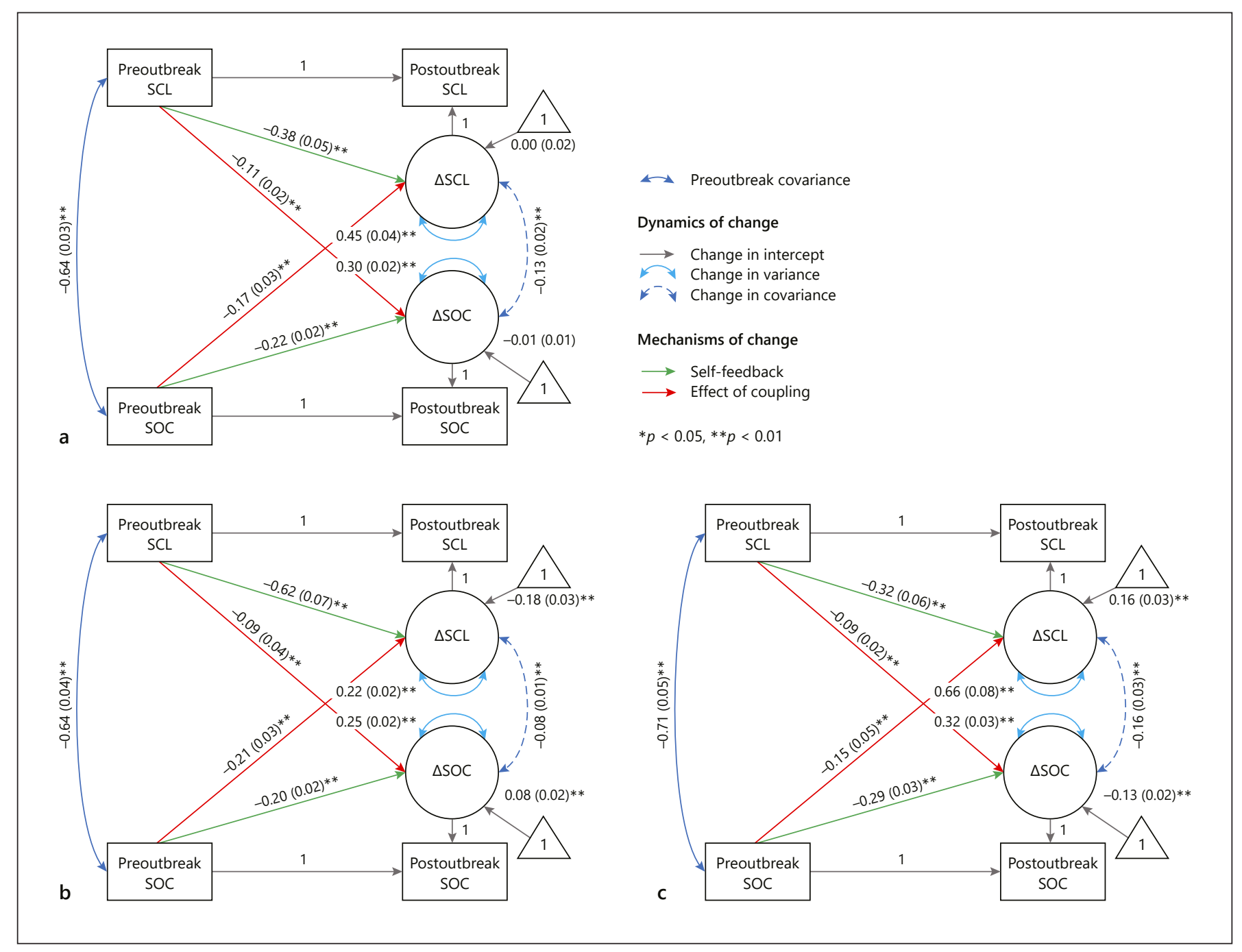

Fig. 2. Estimated parameters for the BLCS model of the relationship between psychopathological symptoms (assessed using the Mini-SCL and SOC). Unstandardized parameters are reported for the total sample $(n=1,591)$ (a) as well as separately for the low- $(n=862)$ (b) and the high-COVID-19-related-stress groups $(n=634)(\mathbf{c})$. b, c Due to missing data for COVID-19-related rumination and traumatic distress, not all respondents could be included in the subgroup analyses.

pre-outbreak symptoms and pre-outbreak SOC levels. The same pattern of results was found in the high-stress group and the low-stress group (Table 1). Across all analyses, higher pre-outbreak SOClevels were associated with smaller changes (i.e., increases and decreases) in symptom levels.

\section{Ability of Psychopathological Symptoms to Predict SOC Changes}

In the total sample, individual changes in SOC were significantly predicted by the pre-outbreak SOC and preoutbreak symptoms. The findings were the same for the high-stress group and the low-stress group (Table 1). Higher pre-outbreak symptom levels were related to smaller changes in SOC levels.

\section{Discussion}

This is the first study to examine mental health before and after the COVID-19 outbreak and potential modulatory effects of SOC. Despite the overall stability (82\%), we identified clinically significant symptom changes in $18 \%$ of respondents (increased in $10 \%$ and decreased in $8 \%$ ). 
Table 1. Results of BLCS modeling

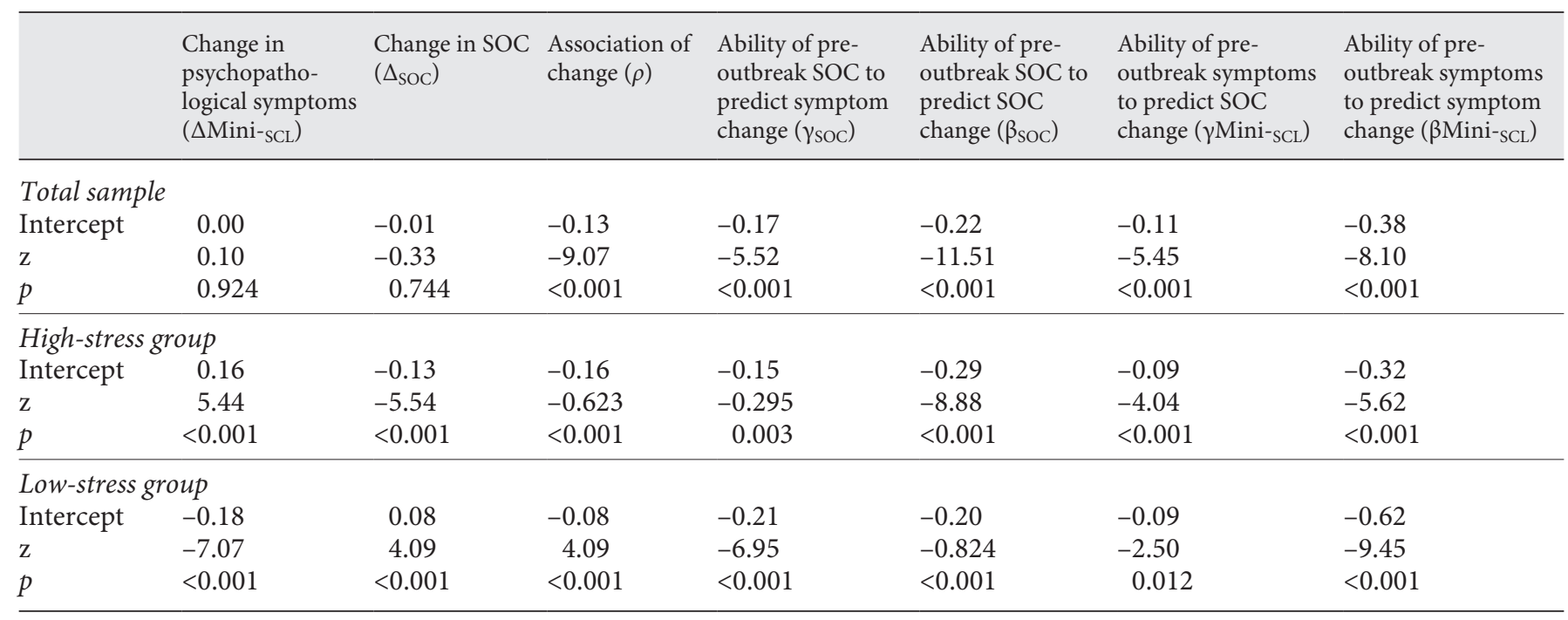

Moreover, 15\% showed above-cut-off COVID-19-related traumatic distress. Taking COVID-19-related stress into account, we identified a group that experienced aboveaverage stress levels and another group that experienced below-average stress levels. While symptoms increased in the high-stress group, the low-stress group showed reduced symptoms at the post-outbreak assessment. Moreover, we consistently identified SOC as a predictor of individual symptom change, with higher SOC levels predicting smaller symptom changes.

In line with previous research, we found stress to be higher in women $[7,8]$ and younger respondents [9]. One may assume that as-of-yet-unknown factors contributed to this observation (e.g., women may be more stressed during lockdown, as they may be overproportionately burdened by childcare duties). Furthermore, corresponding to prior studies $[7,9]$, stress levels were higher in respondents experiencing a poor sleep quality. Interestingly, respondents living in high-risk regions were not more likely to report higher levels of COVID-19-related stress. As such, factors that are associated with a greater psychological vulnerability seem to be more important than factors associated with the risk of infection in predicting mental health consequences of the pandemic.

Beyond insights on risk factors of COVID-19 related psychopathology, the current study sheds further light on the impact of SOC on psychopathology. Our results firstly demonstrate that pre-stressor SOC predicts symptom changes over a short pre- to post-assessment interval. Higher levels of pre-outbreak SOC were related to small- er symptom changes. SOC may thus buffer the impact of stressors on mental health without necessarily resulting in lower symptom levels. Moreover, we did not find differences between the high-stress group and low-stress group concerning the ability of SOC ability to predict symptom changes. Thereby, our findings challenge the assumption that SOC is of particular importance in highstress situations and may thus suggest its universal relevance as a component of euthymia [20,21]. Additionally, our study provides important insights into the temporal stability of SOC. Consistent with the salutogenesis framework [13], we did not find an overall change in SOC levels. However, in line with previous studies challenging the concept of a stable disposition [16,37], we found SOC to be reduced in respondents reporting above-average stress levels. By contrast, those experiencing below-average stress reported increased levels of SOC and decreased symptom levels. No study to date has demonstrated such a steeling effect of stressful life events on SOC [38]. These findings indicate that SOC-targeting trainings $[39,40]$ may be useful to enhance resistance by enabling individuals to buffer negative mental health consequences of stressors.

The current study has the following limitations. First, this study used a nonrepresentative sample (see online suppl. material for details) and was purely observational. We aimed to account for different responses to COVID-19 by conducting subgroup analyses. However, changes in psychopathological symptoms also occur in a percentage of respondents when assessing cohorts over 
time without specific stressors $[41,42]$, which may account for some of the findings. Nevertheless, it is important to note that significant pre- to post-symptom increases were more likely in the high-stress group than in the low-COVID-19-stress group $\left(\chi^{2}[1]=52.87, p<0.001\right)$. This finding strengthens our interpretation. Second, to assess COVID-19-related stress we had to modify wellestablished instruments, thereby affecting cross-study comparability. Third, the time of assessment may not capture the most stressful period of the COVID-19 outbreak but rather a marked change in policymaking in Germany. Thus, we plan to conduct 2 further assessments in the sample, which will also explore the surprising finding of a potential steeling effect, its potential sources (e.g., successful use of coping resources), and why higher preoutbreak psychopathological symptom levels were related to smaller symptom changes.

Despite these limitations, the findings of the current study improve our understanding of the mental health consequences of COVID-19. Despite the majority of respondents showing no clinically significant symptom change, our results demonstrate that a group of respondents characterized by low levels of SOC may be at risk for the development of clinically significant symptom change from pre- to post-outbreak. Future studies should investigate the pandemic's impact on public mental health but also its influence on the mental health of health care professionals $[43,44]$. Our results may also support the development of resilience trainings [45]. Furthermore, future research should address a broad range of psychosocial consequences of COVID-19 [11] and their impact on treatment access for mental disorders [46, 47].

\section{Acknowledgment}

We thank 2 anonymous reviewers for their insightful comments that helped to improve this paper.

\section{Statement of Ethics}

This study was conducted in accordance with the ethical standards of the Ethics Committee of Saarland University and the 1964 Helsinki Declaration and its later amendments or comparable ethical standards. According to the Ethics Committee of Saarland University, this study was exempt from ethical approval requirements.

\section{Conflict of Interest Statement}

This article received no support from any funding agency, commercial business, or not-for-profit institution. In the last 3 years, the authors have had no commercial conflict of interests.

\section{Funding Sources}

None.

\section{Author Contributions}

S.K.S., M.R.S., C.G.S., T.M., and A.S.G. designed and planned this study. A.S.G. collected the data. S.K.S., M.R.S., C.G.S., and M.S. analyzed the data and interpreted the results. S.K.S., M.R.S., M.S., and T.M. wrote this paper. All of the authors reviewed and approved the final version of this paper.

\section{References}

1 Dong E, Du H, Gardner L. An interactive web-based dashboard to track COVID-19 in real time. Lancet Infect Dis. 2020 May;20(5): 533-4.

2 Mak IW, Chu CM, Pan PC, Yiu MG, Ho SC, Chan VL. Risk factors for chronic post-traumatic stress disorder (PTSD) in SARS survivors. Gen Hosp Psychiatry. 2010 Nov-Dec; 32(6):590-8.

3 Mak IW, Chu CM, Pan PC, Yiu MG, Chan VL. Long-term psychiatric morbidities among SARS survivors. Gen Hosp Psychiatry. 2009 Jul-Aug;31(4):318-26.

4 Lung FW, Lu YC, Chang YY, Shu BC. others. Mental symptoms in different health professionals during the SARS attack: a follow-up study. Psychiatr Q. 2009 Jun;80(2):107-16.
5 Rajkumar RP. COVID-19 and mental health: a review of the existing literature. Asian J Psychiatr. 2020 Apr;52:102066.

6 Vindegaard N, Benros ME. COVID-19 pandemic and mental health consequences: systematic review of the current evidence. Brain Behav Immun. 2020. doi: 10.1016/j. bbi.2020.05.048.

7 Liu N, Zhang F, Wei C, Jia Y, Shang Z, Sun L, et al. Prevalence and predictors of PTSS during COVID-19 outbreak in China hardest-hit areas: gender differences matter. Psychiatry Res. 2020 May;287:112921.
8 Wang C, Pan R, Wan X, Tan Y, Xu L, Ho CS, et al. Immediate psychological responses and associated factors during the initial stage of the 2019 coronavirus disease (COVID-19) epidemic among the general population in China. Int J Environ Res Public Health. 2020 Mar;17(5):1729.

9 Huang Y, Zhao N. Mental health burden for the public affected by the COVID-19 outbreak in China: who will be the high-risk group? Psychol Health Med. 2020. doi: 10.1080/13548506.2020.1754438.

10 Holmes EA, O’Connor RC, Perry VH, Tracey I, Wessely S, Arseneault L, et al. Multidisciplinary research priorities for the COVID-19 pandemic: a call for action for mental health science. Lancet Psychiatry. 2020 Jun;7(6): 547-60. 
11 Bavel JJ, Baicker K, Boggio PS, Capraro V, Cichocka A, Cikara M, et al. Using social and behavioural science to support COVID-19 pandemic response. Nat Hum Behav. 2020 May;4(5):460-71.

12 Gordon JA, Borja SE. The COVID-19 Pandemic: Setting the Mental Health Research Agenda [published online ahead of print, 2020 May 18]. Biol Psychiatry. 2020 Jul;88(2): $130-1$.

13 Antonovsky A. Health, Stress, and Coping. San Francisco, CA, US: Jossey-Bass; 1979.

14 Antonovsky A. Unraveling the mystery of health: How people manage stress and stay well. San Francisco, CA, US: Jossey-Bass; 1987.

15 Mittelmark MB, Sagy S, Eriksson M, Bauer G, Pelikan JM, Lindström B, et al., editors. The handbook of salutogenesis [Internet]. Cham: Springer; 2017 [cited 2020 April 22]. Available from: www.springer.com/gp/book/ 978331904599.

16 Volanen SM, Suominen S, Lahelma E, Koskenvuo $M$, Silventoinen K. Negative life events and stability of sense of coherence: a five-year follow-up study of Finnish women and men. Scand J Psychol. 2007 Oct;48(5): 433-41.

17 Eriksson M, Lindström B. Antonovsky's sense of coherence scale and the relation with health: a systematic review. J Epidemiol Community Health. 2006 May;60(5):376-81.

18 Schäfer SK, Becker N, King L, Horsch A, Michael $\mathrm{T}$. The relationship between sense of coherence and post-traumatic stress: a metaanalysis. Eur J Psychotraumatol. 2019 Jan; 10(1):1562839.

19 Del-Pino-Casado R, Espinosa-Medina A, López-Martínez C, Orgeta V. Sense of coherence, burden and mental health in caregiving: a systematic review and meta-analysis. J Affect Disord. 2019 Jan;242:14-21.

20 Fava GA, Bech P. The concept of euthymia. Psychother Psychosom. 2016;85(1):1-5.

21 Fava GA, Guidi J. The pursuit of euthymia. World Psychiatry. 2020 Feb;19(1):40-50.

22 Engelhard IM, van den Hout MA, Vlaeyen JW. The sense of coherence in early pregnancy and crisis support and posttraumatic stress after pregnancy loss: a prospective study. Behav Med. 2003;29(2):80-4.

23 Schäfer SK, Schanz CG, Sopp MR, LassHennemann J, Käfer M, Michael T. Pre-rehabilitation sense of coherence as a predictor of symptom change after rehabilitation. Stress Health. 2020. doi: 10.1002/smi.2949.
24 Kievit RA, Brandmaier AM, Ziegler G, van Harmelen AL, de Mooij SM, Moutoussis M, et al.; NSPN Consortium. Developmental cognitive neuroscience using latent change score models: a tutorial and applications. Dev Cogn Neurosci. 2018 Oct;33:99-117.

25 Mukhtar S. Psychological health during the coronavirus disease 2019 pandemic outbreak [published online ahead of print, 2020 May 21]. Int J Soc Psychiatry. 2020 Aug;66(5): $512-6$.

26 Bonanno GA, Ho SM, Chan JC, Kwong RS, Cheung CK, Wong CP, et al. Psychological resilience and dysfunction among hospitalized survivors of the SARS epidemic in Hong Kong: a latent class approach. Health Psychol. 2008 Sep;27(5):659-67.

27 Göritz AS, Borchert K, Hirth M. Using attention testing to select crowdsourced workers and research participants. Soc Sci Comput Rev. 2019. doi: 10.1177/0894439319848726.

28 Leiner DJ. SoSci Survey. Version 2.5. 00-i1142 [software]. 2019.

29 World Medical Association. World Medical Association Declaration of Helsinki: ethical principles for medical research involving human subjects. JAMA. 2013 Nov;310(20): 2191-4.

30 Singer S, Brähler E. Die "Sense of Coherence Scale«: Testhandbuch zur deutschen Version. Göttingen: Vandenhoeck \& Ruprecht; 2007.

31 Franke G. BSCL-53 ${ }^{\circledR}$-S. Brief SymptomChecklist-Standard - Deutsches Manual. Göttingen: Hogrefe; 2017.

32 Snyder E, Cai B, DeMuro C, Morrison MF, Ball W. A new single-item sleep quality scale: results of psychometric evaluation in patients with chronic primary insomnia and depression. J Clin Sleep Med. 2018 Nov;14(11): 1849-57.

33 Ehring T, Zetsche U, Weidacker K, Wahl K, Schönfeld S, Ehlers A. The Perseverative Thinking Questionnaire (PTQ): validation of a content-independent measure of repetitive negative thinking. J Behav Ther Exp Psychiatry. 2011 Jun;42(2):225-32.

34 Maercker A. Der Peri-Trauma-BelastungsFragebogen - Deutsche Übersetzung. Zürich: Universität Zürich; 2002.
35 Bunnell BE, Davidson TM, Ruggiero KJ. The Peritraumatic Distress Inventory: factor structure and predictive validity in traumatically injured patients admitted through a level I trauma center. J Anxiety Disord. 2018 Apr;55:8-13.

36 Schermelleh-Engel K, Moosbrugger H, Müller $\mathrm{H}$. others. Evaluating the fit of structural equation models: tests of significance and descriptive goodness-of-fit measures. Methods Psychol Res Online. 2003;8(2):23-74.

37 Geyer S. Some conceptual considerations on the sense of coherence. Soc Sci Med. 1997 Jun; 44(12):1771-9.

38 Höltge J, Mc Gee SL, Maercker A, Thoma MV. A salutogenic perspective on adverse experiences. Eur J Health Psychol. 2018;25(2): 53-69.

39 Ando M, Natsume T, Kukihara H, Shibata H, Ito S. Efficacy of mindfulness-based meditation therapy on the sense of coherence and mental health of nurses. Health. 2011;3(2): 118-22.

40 Valtonen M, Raiskila T, Veijola J, Läksy K, Kauhanen ML, Kiuttu J, et al. Enhancing sense of coherence via early intervention among depressed occupational health care clients. Nord J Psychiatry. 2015;69(7):515-22.

41 Wetherell JL, Gatz M, Pedersen NL. A longitudinal analysis of anxiety and depressive symptoms. Psychol Aging. 2001 Jun; 16(2): 187-95.

42 Merikangas KR, Zhang H, Avenevoli S, Acharyya S, Neuenschwander M, Angst J; Zurich Cohort Study. Longitudinal trajectories of depression and anxiety in a prospective community study: the Zurich Cohort Study. Arch Gen Psychiatry. 2003 Oct;60(10):993-1000.

43 Zhang WR, Wang K, Yin L, Zhao WF, Xue Q, Peng M, et al. Mental Health and Psychosocial Problems of Medical Health Workers during the COVID-19 Epidemic in China. Psychother Psychosom. 2020;89(4):242-50.

44 Theorell T. COVID-19 and Working Conditions in Health Care. Psychother Psychosom. 2020;89(4):193-4.

45 Fava GA, Cosci F, Sonino N. Current Psychosomatic Practice. Psychother Psychosom. 2017;86(1):13-30.

46 Wright JH, Caudill R. Remote Treatment Delivery in Response to the COVID-19 Pandemic. Psychother Psychosom. 2020;89(3):130-2.

47 Zhai Y. A call for addressing barriers to telemedicine: health disparities during the $\mathrm{CO}$ VID-19 pandemic. Psychother Psychosom. 2020. doi: $10.1159 / 000509000$. 